\title{
Towards the structural analysis of an F plasmid protein, TraW Christina Rodriguez ${ }^{1}$ \\ ${ }^{1}$ York University \\ csr07@yorku.ca
}

Bacterial conjugation is a form of lateral gene transfer used in bacterial systems to allow for the transfer of genetic material by a contact dependent mechanism. This mechanism has been considered to greatly contribute to the increase of virulence factors among different pathogens. This transmission of conjugative plasmids can be achieved by the type IV secretion system (T4SS). The T4SS is a multiprotein complex spanning the inner and outer membrane of gram-negative bacteria, producing a pilus to allow contact with neighbouring cells. The T4SS is able to produce many different types of pili, the focus of this study is on the F pilus. The F pilus is thin, flexible and capable of extension and retraction. The F pilus is composed of TraA pilin monomers, and the intricate process of assembling these monomers is achieved by many different proteins including TraW. TraW is a protein that is specific to the F-type T4SS and is present in the periplasmic space. TraW is essential for the assembly and extension of the pilus as well as bacterial conjugation. Mutations in TraW stop the pilus from extending. While the interaction between the N-terminal domain of TraW and the $\mathrm{C}$-terminal domain of TrbC is essential for conjugation to move forward. Although the general function of this protein is understood the intricate process required to achieve them is not, understanding this protein at a structural level will provide better insight into the role this protein plays within the T4SS. 